\title{
Opto-Mechanical Manipulation of Stem Cells
}

\author{
Hu Zhang, Kuo-Kang Liu* and Alica El Haj
}

Institute of Science and Technology in Medicine, Keele University, Stoke-on-Trent, ST4 7QB, UK

\begin{abstract}
Stem cells have a great potential for regeneration of injured or degenerated tissues or organs. In delivery of cell therapy, stem cells are normally subjected to different levels of shear stress which may have a great impact on their proliferation and differentiation after they reach the target site. Although the shear stress has been recognized as one of key factors, the study on how it affects single stem cell mechanobiologically is not available. This paper reports an application of optical tweezers for manipulating the human mesenchymal stem cells which are exposed to various levels of shear stress in flow chambers. Our preliminary results show different responses of stem cells (the tether formation, cell shrinkage, presence of cell surface nanoparticles, cell rotation before aligning the flow direction) under shear forces in different flow chambers. Also, a few interesting optical characteristics of stem cells have been noticed; for example, the variation of refractive index within the cell may lead to different behaviors when they are manipulated by optical tweezers.
\end{abstract}

Keywords: Mesenchymal stem cells, optical tweezers, shear stress, manipulation, single cell, variation.

\section{INTRODUCTION}

Human mesenchymal stem cells (hMSCs) are pluripotent cells which have the capacity to differentiate along osteogenic, chondrogenic and adipogenic lineages [1]. Those cells hold enormous potentials for treatments and ultimately for cures of many diseases for which the adequate therapies do not exist. Before progressing into clinical applications, many practical issues need to be addressed so that function and structural integrity of stem cells can be maintained through extraction and isolation from patients, in-vitro proliferation, injection and delivery into patient bodies. More importantly, when stem cells reach the targeted sites, those cells must be able to differentiate into the specific tissues as required. It is essential to investigate effects of the mechanical factors during injection, delivery and circulation of stem cells within human bodies on the properties of stem cells.

It has been reported that a number of mechanical forces are important biological stimuli on the stem cells [2]. Fluid velocities as low as $\sim 0.1-1.0 \mu \mathrm{m} / \mathrm{s}$ have considerable influence on the cell microenvironment and in modulating morphogenesis [3]. An increase in mineral deposition by marrow stromal osteoblasts was found in the shear stress range of 0.01 to $0.03 \mathrm{~Pa}$ in a transverse flow bioreactor system. The expansion and differential phenotype of osteoblasts grown in bioreactor systems were enhanced when shear stress was in the range of $0.5-1.5 \mathrm{~Pa}$ in 2-D planar plates [49]. In 3-D constructs, however, an average surface stress of $5 \times 10^{-5} \mathrm{~Pa}$ was found to correspond to increased cell viability and proliferation whereas higher shear stresses of $1 \times 10^{-3} \mathrm{~Pa}$ was associated with up-regulation of mRNA expression of Runx2, osteocalcin, and alkaline phosphatase [10,11]. Zhao et al. [12] found that in a perfusion bioreactor, a 1.4 times higher proliferation rate, higher colony forming unit-

*Address correspondence to this author at the Institute of Science and Technology in Medicine, Keele University, Stoke-on-Trent, ST4 7QB, UK; Tel: 44(0)-1782-554600; Fax: 44(0)-1782-717709; E-mail: i.k.liu@ @eele.ac.uk fibroblast (CFU-F) formation, and more fibronectin and heat shock protein (HSP)-47 secretion were observed for hMSCs at lower flow rate corresponding to interface shear stress of $0.1 \times 10^{-4} \mathrm{~Pa}$, however, higher flow rate corresponding to initial interface shear stress of $1.2 \times 10^{-4} \mathrm{~Pa}$ up-regulated osteogenic differentiation of hMSCs. Majority of reports have been focused on the effect of shear stress on the monolayer spreading hMSCs during in-vitro 2-D or 3-D cultivation, how hMSCs respond to shear stress in physiological flow remains unknown. Especially how an individual cell responds to different levels of shear stress and the various responses of different single cells will be a key issue for clinical applications of hMSCs.

Although the shear stress has been recognised as one of key factors which may have a great impact on the differential capacities of stem cells, lack of quantitative manipulation tools posts a significant hindrance to study the mechanical effect on single cells. Recently, optical tweezers have emerged as an essential tool for manipulating single biological cells and performing sophisticated biophysical/ biomechanical characterizations. Distinct advantages of using tweezers for these characterizations include non-contact force for cell manipulation, force resolution as accurate as $100 \mathrm{aN}$, and amiability to liquid medium environments [13]. Optical tweezers have been widely employed as a tool for actively manipulating and positioning biological objects at nano-/micro-scale. One of the most popular applications is to apply tweezers to confine or constrain single cells, as well as to stimulate, organize, assemble, locate, sort and modify them $[14,15]$. In this study, we employed optical tweezers to exert a shear stress on a single stem cell, and the observation results of manipulating stem cells are presented.

\section{MATERIALS AND METHODOLOGY}

\section{Optical Tweezers}

The commercial optical tweezers (Cell Robotic, Inc, USA) which was driven by a Windows XP based software was used in the present experimental work. The source of 
laser was Nd:YAG at a wavelength of $1064 \mathrm{~nm}$ pumped by a $1.5 \mathrm{~W}$ diode. Laser was then reflected through dichroic mirrors and focused through an inverted microscope (Nikon optical microsystem) before it reached the objects.

\section{Operational Chambers}

Two different chambers have been used for the experimental operation. A Lab-Tek chamber (Nunc Inc., Naperville, IL) without coating was initially used for manipulation of hMSCs. The CoverWell perfusion chambers (Grace Biolabs, OR, USA) were coated with different chemical reagents. Three different coating methods have been used for preventing adherence of stem cells on the surfaces of glass substrates. The first coating method was to utilize dipcoating the thin glass $(1 \mathrm{~mm})$ into a $1 \% \mathrm{w} / \mathrm{v}$ solution of agarose (Sigma) at $65^{\circ} \mathrm{C}$ and drying overnight at room temperature. The second coating method was to incubate cover glass in a mixture of $1 \mathrm{~mL}$ of aminopropyltriethoxysilane (Sigma, Dorset, UK) and $50 \mathrm{~mL}$ acetone for 20 seconds, wash twice in acetone and twice in deionized water, and dry at room temperature. The third coating method was to dissolve 40mg poly(2-hydroxyethylmethacrylate) (Sigma, Dorset, UK) in $2 \mathrm{~mL} 95 \%$ ethanol, apply dip-coating the thin cover glass into the solution, and dry overnight at room temperature.

\section{Cells and Micro-Bead Attached Cells}

hMSCs of a male patient were obtained from Lonza (Lonza Walkersville, MD, USA). Cells of passage 4 were stocked in liquid nitrogen. The cells were re-suspended in cell culture media (10\% fetal calf serum (FCS), $1 \%$ antibiotics, and 1\% L-glutamine in Dulbecco's Modified Eagle's Medium (DMEM) solution (low glucose)). The cells were placed in 24 -well plates and were maintained at $37^{\circ} \mathrm{C}$ in $5 \%$ $\mathrm{CO}_{2}$. The media was changed every two days and the cells were monitored until cells became confluent. The cells were detached using trypsin-EDTA. After centrifugation, cells were re-suspended in serum-free media. The cells of passage 5 were used for all experiments described here. The force exerting on a single cell was calculated from the Stokes law, $\mathrm{F}=6 \pi \mathrm{r} \eta \mathrm{u}$, where $\mathrm{r}$ was the cell radius, $\eta$ was the liquid viscosity and $u$ was the flow velocity. The shear stress was obtained by the force over the cross-section area of the single cell.

To prepare micro-bead attached cells, the cell media was removed from the cell culture wells, and cells were washed with sterile phosphate buffered saline (PBS) solution. $2 \mathrm{~mL}$ of serum free media and Arg-Gly-Asp (RGD) (Sigma, Dorset, UK) coated magnetic particle $\left(4.5 \mu \mathrm{m} \mathrm{CrO}_{2}\right.$ particle,

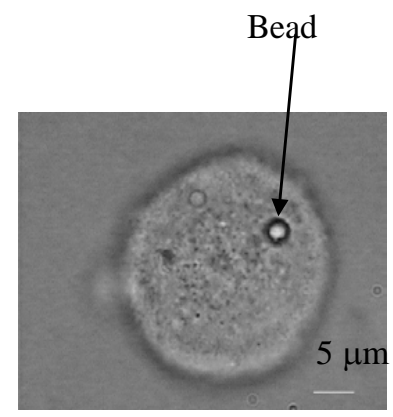

(a)
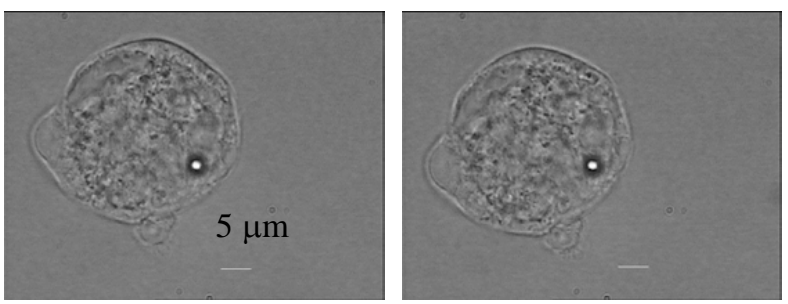

(b)
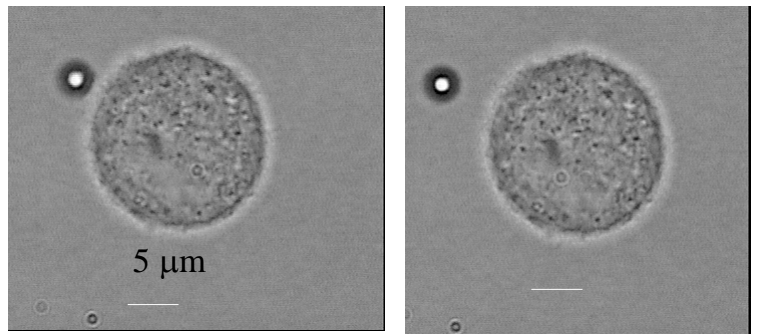
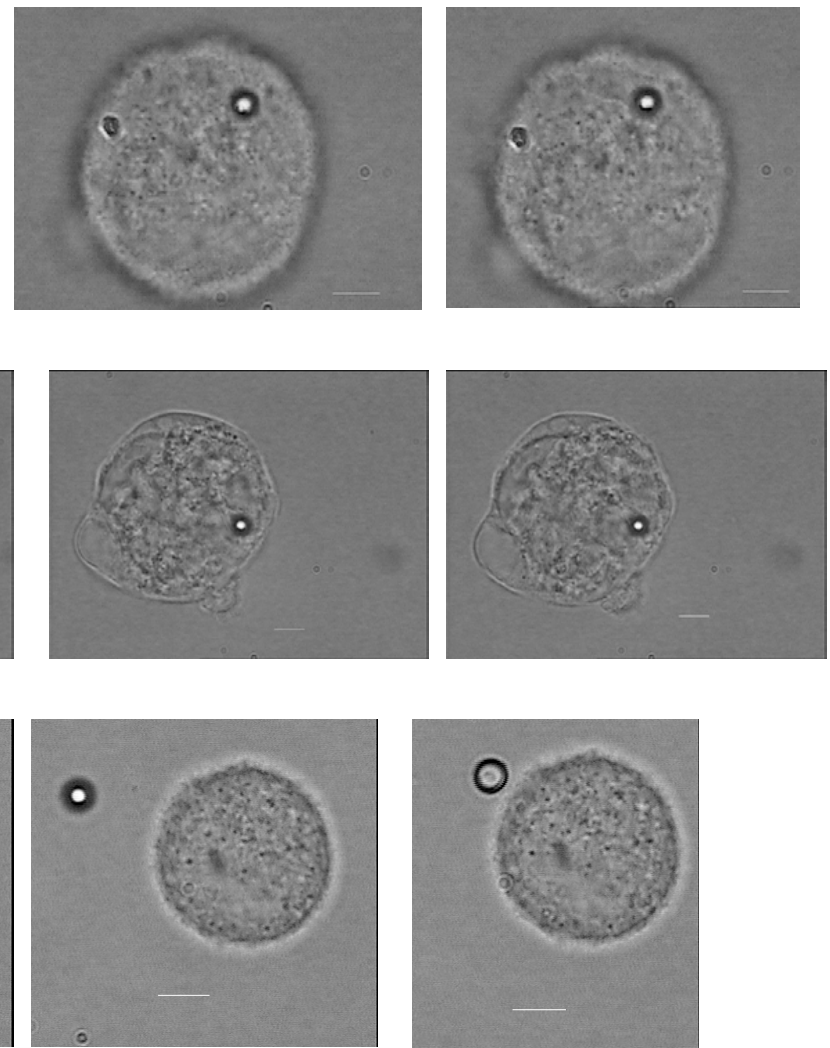

(c)

Fig. (1). Manipulation of stem cells in an uncoated flow chamber. (a) The bead-attached stem cell moved in PBS solution at $40 \mu \mathrm{m} / \mathrm{s}$ (corresponding to the shear stress of $0.024 \mathrm{~Pa}$ ) and the flow direction was from the left to right. (b) The bead-attached stem cell rotated when optical force dragged the cell at $40 \mu \mathrm{m} / \mathrm{s}$ in PBS solution. The flow direction is from the left to right. (c) The bead-attached stem cell was fully attached to the bottom glass in PBS solution. The optical force tried to move the cell at $40 \mu \mathrm{m} / \mathrm{s}$, the bead was moved away from the cell membrane, but bounced back to cell membrane again. The tether formation between the bead and the cell can be used to measure the cell membrane mechanics. Scale Bar: $5 \mu \mathrm{m}$. 
Spherotech Inc.) suspension (the ratio between particles to cells was around 1.5:1) were added into each well. The wells were placed back to the incubator for $40 \mathrm{~min}$. The media was removed and the cells were washed with PBS solution and harvested for further experiments.

\section{RESULTS AND DISCUSSION}

\section{Uncoated Operational Chambers}

hMSCs were suspended in a glass container, however, when they were transferred to liquid chambers, majority of cells settled down to the bottom glass surface. Fig. (1) shows that bead-attached hMSCs floated, partially attached, and fully attached in the uncoated operational chambers. The cell was displayed nearly spherical when floating (Fig. 1a). It was captured by the optical forces and fixed in the optical trap. The stage moved at different rates to create a relative flow environment on the cell. The cell could be deformed as it was exposed to certain levels of shear stress. However, as the cell had a cytoskeleton and other intracellular materials, the extent of deformation was so minor at the given flow conditions that accurate deformation data were difficult to quantify.

When a cell was partially attached to the bottom (Fig. 1b), the cell was driven to rotate around its attached site. Most of cells, however, were fully attached to the bottom. Those cells were not able to move in the chamber. In Fig. (1c), the bead was moved away from the cell surface as the optical force was greater than the adherent force between the bead and the cell, but after a certain distance, the bead was bounced back to the cell surface. The tether formation between the cell surface and the bead has been used to quantitatively characterize the stem cell membrane mechanics [16]. A quite large bead was used in this study and the tether formed between the bead and the cell surface was estimated to be $8.6 \mu \mathrm{m}$, smaller than the averaged tether length of 10.6 $\mu \mathrm{m}[16]$.

\section{Coated Operational Chambers}

To prevent adherence of stem cells on the glass surface, three different means have been used to coat the operational chambers. Cells could only suspend for less than $5 \mathrm{~min}$ and then settle down to bottom using either agarose or aminopropyltriethoxysilane coated chambers. However, poly (2hydroxyethylmethacrylate) formed a quite thin film on the surface and this film prevented cell attaching to the bottom. hMSCs were able to float at different flow rates $(10 \sim 100$ $\mu \mathrm{m} / \mathrm{s}$, corresponding to shear stress of $0.006 \sim 0.06 \mathrm{~Pa}$ ). During manipulation of those hMSCs, varieties in shape, size and response to shear stress were observed for current stem cells without further isolating the subpopulation.

Microspikes on the stem cell membrane surface similar in structure to a glycocalyx found on endothelial cells have been observed during manipulation as shown in Fig. (2a). The presence of the surrounding structures leads to a tendency for cells to adhere strongly to their surrounding surface or neighboring cells. The optical force was strong enough to break the adherent force so the cell in Fig. (2a) was able to move at a flow rate of $40 \mu \mathrm{m} / \mathrm{s}$ (equivalent shear stress of $0.02 \mathrm{~Pa}$ ). However, after 20 seconds, the microspikes on the membrane surface disappeared and the cell shrank to $70 \%$ of its original size (reducing from $30.1 \mu \mathrm{m}$ to $20.5 \mu \mathrm{m})$ as shown in Fig. (2b) when it was dragged away for continuous movement. Although there are no previous reports on glycocalyx structures on marrow derived stem cells, this rapid response to fluid shear stress is a known feature of endothelial cells and their glycocalyx when exposed to fluid shear [17].

It was also interesting to observe that the laser light struck on certain part of the cell and the force acting on the part trapped the cell to move in the liquid chamber. When the light struck on the other sites, the cell started to rotate until light struck on the correct part of the cell and then it moved smoothly, as shown in Fig. (3). When light struck the

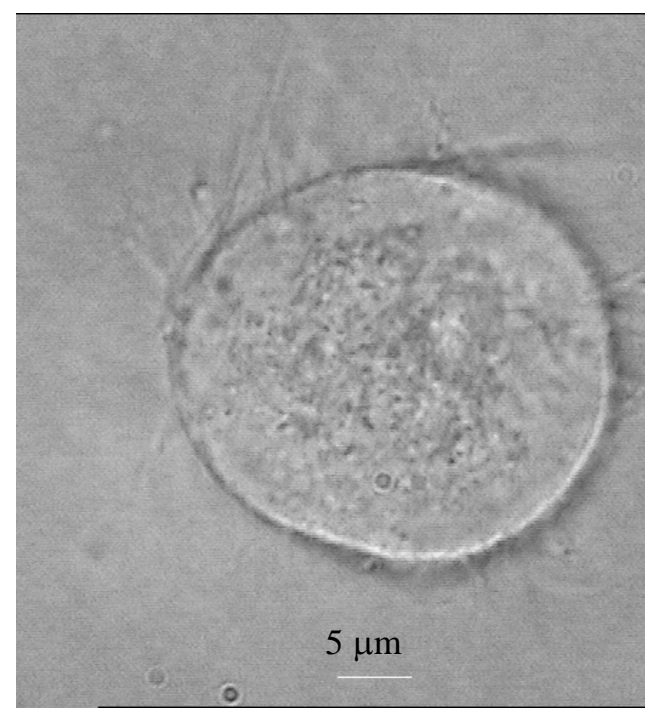

(a)

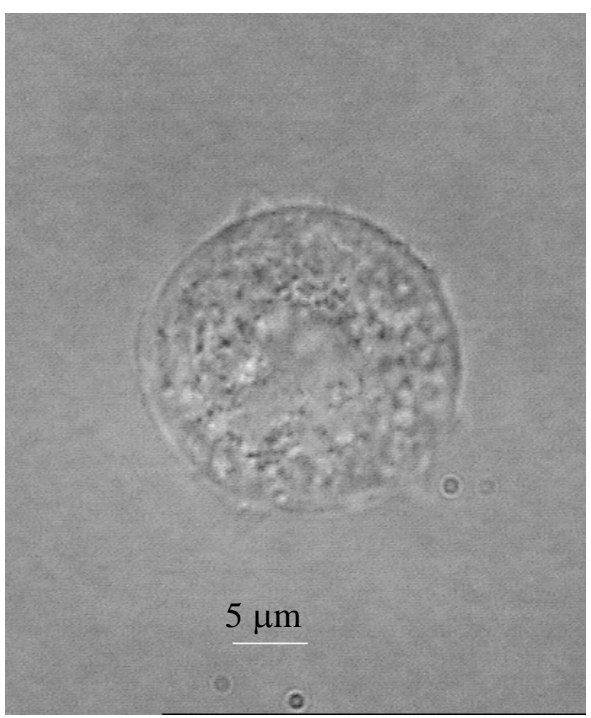

(b)

Fig. (2). Shape change of stem cells subjected to a flow rate of $40 \mu \mathrm{m} / \mathrm{s}$ (corresponding to the shear stress of $0.02 \mathrm{~Pa}$ ). (a) Microspikes were observed around the cell membrane surface. The cell with microspikes was dragged to move in the serum-free media for $1 \mathrm{~mm}$. (b) After moving the cell for $1 \mathrm{~mm}$, the microspikes of stem cell disappeared and the cell shrank to reduce the shear damage. Scale Bar: $5 \mu \mathrm{m}$. 


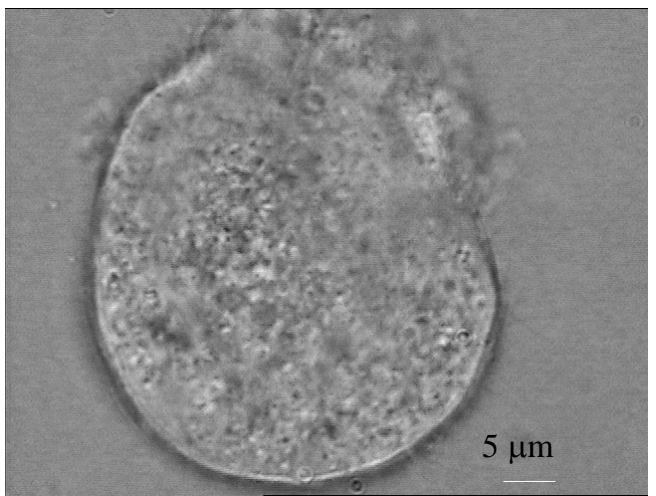

(a)

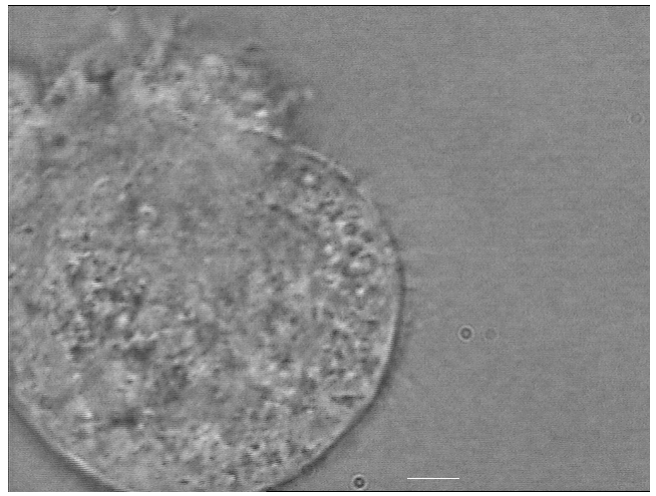

(c)

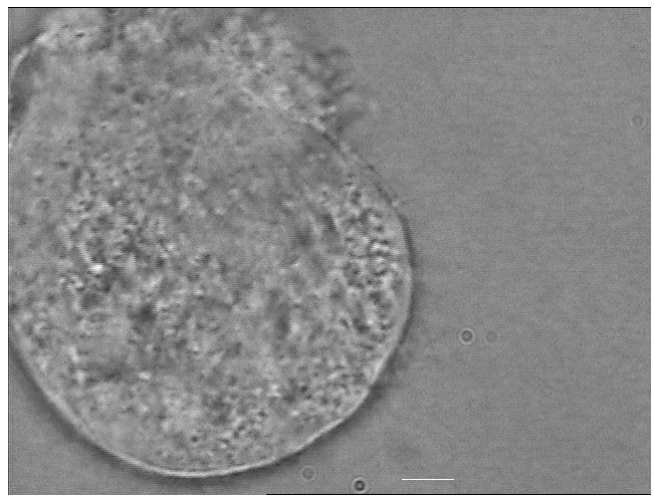

(b)

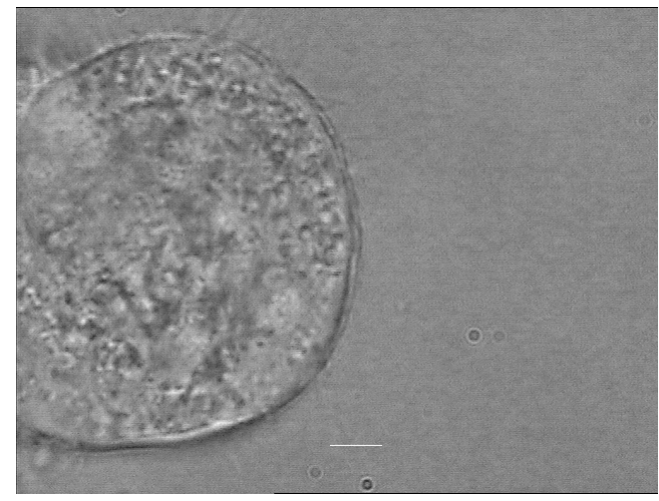

(d)

Fig. (3). The stem cell was trapped by optical tweezers and moved from left to right. The stem cell rotated itself from (a) to (d) and the final shape (d) was kept to move in the fluid flow. Scale Bar: $5 \mu \mathrm{m}$.

central opaque region, the cell was pushed away from the optical trap as shown in Fig. (4). It has been reported that different intracellular organelles have different refractive indices. Organelles of higher refractive index can be trapped and moved while those of lower refractive index than their surrounding were repelled by the trap [18]. Fig. (3 and 4) confirmed that stem cells have organelles with higher or lower refractive index which behaved dramatically different when trapped by the optical tweezers.

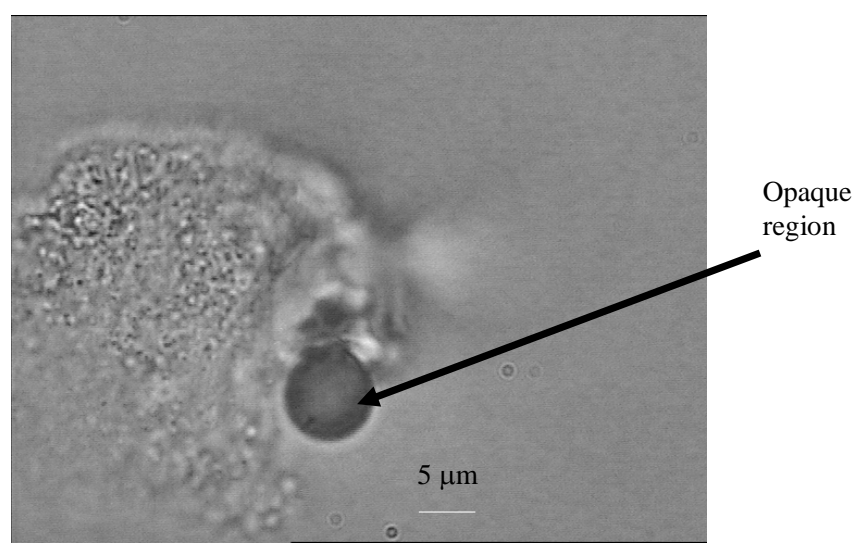

Fig. (4). The optical tweezers focused on the cell and dragged it to move in the liquid media. When the light struck on the opaque region, the cell was expelled from the optical trap. Scale Bar: $5 \mu \mathrm{m}$
It was observed that there were nano-scale vesicles or particles on the cell surface. Release of extracellular membrane particles carrying the stem cell marker prominin- 1 has previously been observed from neural progenitors and other epithelial cells [19] and it has been concluded that the release of membrane particles may have a role in tissue development and maintenance. In our experiments, the laser light was focused on the external particles resulting in the cells being dragged in the liquid media. Some of external particles were removed during the stage movement as shown in Fig. (5). During the initial detachment, the external particles were resistant to the drag force. As the optical force was far greater than the force between the external particles and the stem cell surface, the particle was finally taken away from the surface. This demonstrates how optical tweezers may be ied to investigate the impact of removal of membrane par:les on the characteristics and functions of stem cells.

\section{CONCLUSIONS}

The optical tweezers have been used to trap the human mesenchymal stem cells and move cells at different flow rates in uncoated and coated flow chambers. Poly (2hydroxyethylmethacrylate) coated flow chambers prevented adherence of stem cell on the bottom glass. Responses of stem cells under different levels of shear forces in flow chambers were observed, including the tether formation, cell shrinkage, presence of cell surface nanoparticles, cell rotation before aligning the flow direction. The variation of refractive index within the cell may lead to different behaviors when they are manipulated by optical tweezers. 


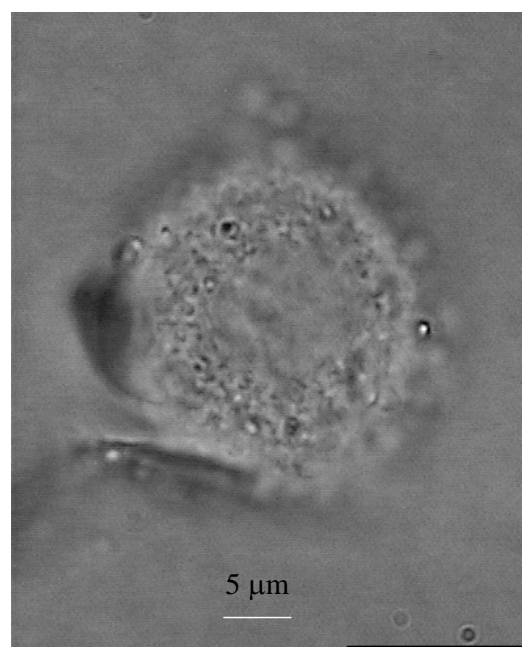

(a)

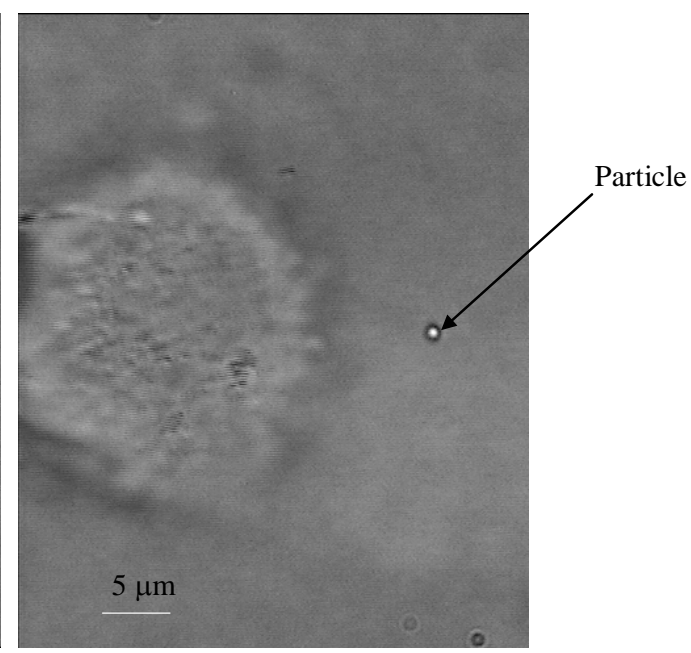

(b)

Fig. (5). Removal of membrane particles from the stem cell by optical forces. (a) The particle was trapped by the optical tweezers. The cell was dragged to flow in the serum free media, and the particle was removed gradually during the movement. (b) The membrane particle was removed away from the stem cell membrane surface. Scale Bar: $5 \mu \mathrm{m}$.

\section{ACKNOWLEDGEMENTS}

The work is supported by the project funding (BB/D014786/1), which is co-funded by BBSCR and EPSRC (Life Science Interface Programme).

\section{REFERENCES}

[1] Jiang Y, Jahagirdar BN, Reinhardt RL, et al. Pluripotency of mesenchymal stem cells derived from adult marrow. Nature 2002; 418: 41-9. Available at: http: //www.nature.com/nature/journal/v418/ n6893/full/nature00870.html - a1

[2] Thomas G, El Haj AJ. Bone marrow stromal cells are load responsive. Calcif Tissue Int 1996; 58(2): 101-8.

[3] Griffith LG, Swartz MA. Capturing complex 3D tissue physiology in vitro. Nat Rev Mol Cell Biol 2006; 7: 211-24.

[4] Hillsley MV, Frangos JA. Alkaline phosphatase in osteoblasts is downregulated by pulsatile fluid flow. Calcif Tissue Int 1997; 60: 48-53.

[5] Jiang GL, White CR, Stevens HY, Frangos JA. Temporal gradients in shear stimulate osteoblastic proliferation via ERK $1 / 2$ and retinoblastoma protein. Am J Physiol Endocrinol Metab 2002; 283: E383-9.

[6] Klein-Nulend J, Helfrich MH, Sterck JG, et al. Nitric oxide response to shear stress by human bone cell cultures is endothelial nitric oxide synthase dependent. Biochem Biophys Res Commun 1998; 250: 108-14.

[7] McAllister TN, Du T, Frangos JA. Fluid shear stress stimulates prostaglandin and nitric oxide release in bone marrowderived preosteoclast-like cells. Biochem Biophys Res Commun 2000; 270: 643-8.

[8] Reich KM, Frangos JA. Effect of flow on prostaglandin E2 and inositol trisphosphate levels in osteoblasts. Am J Physiol Cell Physiol 1991; 261: C428-32.
[9] Smalt R, Mitchell FT, Howard RL, Chambers TJ. Induction prostaglandin E2 in osteoblasts by wall-shear stress but not strain. Am J Physiol Endocrinol Metab 1997; 273: E751-8.

[10] Cartmell SH, Porter BD, Garcia AJ, Guldberg RE. Effects of medium perfusion rate on cell-seeded three-dimensional bone constructs in vitro. Tissue Eng 2003; 6: 1197-203.

[11] Porter B, Zauel R, Stockman H, Guldberg R, Fyhrie D. 3-D computational modeling of media flow through scaffolds in a perfusion bioreactor. J Biomech 2005; 38: 543-9.

[12] Zhao F, Chella R, Ma T. Effects of shear stress on 3-D human mesenchymal stem cell construct development in a perfusion bioreactor system: experiments and hydrodynamic modeling. Biotech Bioeng 2007; 96: 584-95.

[13] Grier DG. A revolution in optical manipulation. Nature 2003; 424 810-6.

[14] Zhang H, Liu KK. Optical tweezers for single cells. J R Soc Interface 2008; 5: 671-90.

[15] Walker LM, Holm A, Cooling L, et al. Mechanical manipulation of bone and cartilage cells with optical tweezers. FEBS Lett 1999; 459(1): 39-42.

[16] Titushkin I , Cho M. Distinct membrane mechanical properties of human mesenchymal stem cells determined using laser optical tweezers. Biophys J 2006; 90: 2582-91.

[17] Savery MD, Damiano ER. The endothelial glycocalyx is hydrodynamically relevant in arterioles throughout the cardiac cycle. Biophys J 2008; 95: 1439-47.

[18] Burnham DR, Wright GD, Read ND, McGloin D. Holographic and single beam optical manipulation of hyphal growth in filamentous fungi. J Opt A 2007; 9: S172-9.

[19] Marzesco AM, Janich P, Wilsch-Brauinger M, et al. Release of extracellular membrane particles carrying the stem cell marker prominin-1 (CD133) from neural progenitors and other epithelial cells. J Cell Sci 2005; 118(13): 2849-58

(C) Zhang et al.; Licensee Bentham Open.

This is an open access article licensed under the terms of the Creative Commons Attribution Non-Commercial License (http://creativecommons.org/licenses/by-nc/3.0/) which permits unrestricted, non-commercial use, distribution and reproduction in any medium, provided the work is properly cited. 